\title{
The ABC Transporter MgAtr4 Is a Virulence Factor of Mycosphaerella graminicola that Affects Colonization of Substomatal Cavities in Wheat Leaves
}

\author{
loannis Stergiopoulos, Lute-Harm Zwiers, and Maarten A. De Waard \\ Laboratory of Phytopathology, Department of Plant Sciences, Wageningen University, Binnenhaven 5, 6709 PD, \\ P.O. Box 8025, 6700 EE Wageningen, The Netherlands
}

Submitted 16 September 2002. Accepted 11 April 2003.

The role in virulence of the ATP-binding cassette (ABC) transporters MgAtr1, MgAtr2, MgAtr3, MgAtr4, and MgAtr5 from Mycosphaerella graminicola was analyzed by gene disruption or replacement on seedlings of the susceptible wheat cultivar Obelisk. Disruption strains of MgAtr1 and MgAtr2 and replacement strains of $M g A t r 3$ and $M g A t r 5$ displayed the same phenotype as control strains, while virulence of the MgAtr4 disruption strains was significantly reduced. This reduction in virulence was independent of the wheat cultivar used. Histopathological analysis of the infection process revealed that $\mathrm{MgAtr} 4$ disruption strains colonize substomatal cavities less efficiently and display reduced intercellular growth in the apoplast of wheat leaves. In vitro growth experiments in different media showed no fitness penalty associated with the disruption of MgAtr4. Expression analysis demonstrated that transcripts of the constitutively expressed gene CYP51 encoding the fungal-specific cytochrome P450 sterol $14 \alpha-$ demethylase from $M$. graminicola were not detectable in interaction RNA from wheat infected with MgAtr4 disruption strains, thus confirming the reduced intercellular growth of these strains. The results indicate that MgAtr4 is a virulence factor of $M$. graminicola during pathogenesis on wheat and may function in protection against fungitoxic compounds present around the substomatal cavities of wheat leaves. MgAtr4 is the first virulence factor cloned from this important plant pathogen.

Additional keywords: compatible interaction, incompatible interaction, pathogenicity, plant defense compounds, Septoria tritici, toxins.

The ascomycete fungus Mycosphaerella graminicola (Fuckel) J. Schröt. in Cohn (anamorph: Septoria tritici Roberge in Desmaz.) is the causal agent of septoria tritici blotch on wheat. This is one of the most important economic diseases of wheat, with potential yield losses of up to 30 to $40 \%$ (Eyal et al. 1987). Typical symptoms of the disease are irregular chlorotic areas on the infected leaves that develop into necrotic lesions covered with dark brown to black pycnidia or pseudothecia of the fungus (Eyal et al. 1987). Conditions of high humidity and moderate temperatures are known to favor the disease.

Histopathological analysis of the infection process showed

Corresponding author: M. A. De Waard; Telephone: +31 317 483123; Fax.: +31 317 483412; E-mail: maarten.dewaard@wur.nl. that the fungus primarily enters its host directly through stomata. Penetration of the substomatal cavities usually occurs within 24 to $48 \mathrm{~h}$ after inoculation and, subsequently, growth extends to the leaf apoplast and to neighboring substomatal cavities, thus establishing multiple stomatal infections from a single penetration site. During pathogenesis, infection hyphae remain strictly intercellular and in close contact with mesophyll cells, but no haustoria or other specialized feeding structures are formed. The first symptoms on infected plants become visible at 8 to 10 days postinoculation (dpi) as chlorotic areas on the leaf surface. From this point onward, there is a massive increase of fungal biomass, presumably induced by the release of nutrients after cell collapse. This increase is especially apparent around the substomatal cavities, where the formation of the so-called baskets is observed. At approximately $14 \mathrm{dpi}$, chlorotic areas turn into necrotic lesions, and the first pycnidia become visible, emerging from stomata with mycelial baskets (Cohen and Eyal 1993; Duncan and Howard 2000; Kema et al. 1996).

Incompatible responses in the wheat- $M$. graminicola interaction are described as the inability of the fungus to grow within its host. Although no differences are observed during the first stages of the infection process, only very little increase in fungal biomass is observed during the later stages of pathogenesis, as the fungus is unable to colonize the mesophyll and remains restricted to the substomatal cavities (Kema et al. 1996). Incompatible interactions can still result in necrosis and production of pycnidia but considerably less as compared with that in compatible interactions. The quantitative differences in response between compatible and incompatible interactions led to the suggestion that no complete immunity to $M$. graminicola exists. In such cases, any restriction or delay in pathogen development can be regarded as a form of resistance (Nelson and Marshall 1990).

Although a considerable amount of data is present regarding epidemiology of infections caused by $M$. graminicola, the biochemical and molecular basis of virulence of the fungus is poorly understood, and little is known about actual genes involved in host resistance and virulence of the pathogen. Recent data suggest a gene-for-gene relationship in the wheat- $M$. graminicola interaction, and studies on the segregation of avirulence led to the identification of a single avirulence locus (Brading et al. 2002; Kema et al. 2000). However, specific avirulence genes have not yet been identified from this pathogen.

ATP-binding cassette (ABC) transporters compose one of the largest protein families known to date. They are present in a wide variety of organisms, ranging from bacteria to man (Higgins 1992). ABC transporters are located in the outer 
plasma membrane or in membranes of intracellular compartments and are capable of transporting a wide variety of hydrophobic compounds against a concentration gradient. The energy needed for transport is generated by ATP hydrolysis and, for this reason, they are considered as primary active transport systems. In filamentous fungi, $\mathrm{ABC}$ transporters can function in protection against synthetic toxic compounds or antibiotics produced by competing microorganisms (Schoonbeek et al. 2002). Overexpression of ABC transporters can have pleiotropic effects, resulting in simultaneous resistance to a number of chemically unrelated compounds, a phenomenon described as multidrug resistance (MDR). In plant pathogenic fungi, $\mathrm{ABC}$ transporters also can act as virulence factors, if they provide protection against plant defense compounds during pathogenesis or mediate secretion of pathogenicity factors, such as host-specific toxins (De Waard 1997; Stergiopoulos et al. 2002b). So far, a role of ABC transporters in pathogenesis has been demonstrated for the plant pathogens Magnaporthe grisea (Urban et al. 1999), Botrytis cinerea (Schoonbeek et al. 2001), and Gibberella pulicaris (Fleissner et al. 2002).

Five ABC transporter genes, coded MgAtrl, MgAtr2, MgAtr3, MgAtr4, and MgAtr5, have been cloned and characterized from M. graminicola (Stergiopoulos et al. 2002a; Zwiers and De Waard 2000). All genes are highly homologous to other fungal $\mathrm{ABC}$ transporter genes involved in MDR or pathogenesis. Heterologous expression in Saccharomyces cerevisiae demonstrated that the encoding proteins transport a wide variety of natural and synthetic toxic compounds. Functional analysis of MgAtrl to MgAtr5 disruption or replacement strains showed that they also provide protection against fungicides and secondary plant metabolites in $M$. graminicola (Zwiers et al., in press).

In this paper, we report the characterization of the disruption or replacement strains of $M g A t r 1$ to $M g A t r 5$, with respect to virulence on wheat. We demonstrate that MgAtr4 disruption strains are significantly less virulent on wheat seedlings as compared with control strains, indicating that MgAtr4 is a virulence factor from $M$. graminicola.

\section{RESULTS}

Virulence assays.

Virulence of all disruption or replacement strains of MgAtrl to MgAtr5 from M. graminicola (Table 1) was com-

Table 1. Strains of Mycosphaerella graminicola tested in virulence assays

\begin{tabular}{|c|c|c|}
\hline Strain name & $\begin{array}{c}\text { Gene disrupted } \\
\text { or replaced }\end{array}$ & $\begin{array}{c}\text { Transformation } \\
\text { method }\end{array}$ \\
\hline $\mathrm{IPO} 23^{\mathrm{a}}$ & - & - \\
\hline $\mathrm{Sp} 2^{\mathrm{b}}$ & - & A. tumefaciens ${ }^{\mathrm{c}}$ \\
\hline $\mathrm{O} 4$ & MgAtrl & A. tumefaciens \\
\hline $\mathrm{O} 8$ & MgAtrl & A. tumefaciens \\
\hline $\mathrm{T} 1$ & MgAtr2 & A. tumefaciens \\
\hline $\mathrm{T} 2$ & MgAtr2 & A. tumefaciens \\
\hline 58 & MgAtr3 & $\mathrm{PEG}^{\mathrm{d}}$ \\
\hline 85 & MgAtr3 & PEG \\
\hline$\Delta \mathrm{Mg} \operatorname{Atr} 4.3$ & MgAtr4 & A. tumefaciens \\
\hline$\Delta \mathrm{MgAtr} 4.4$ & MgAtr4 & A. tumefaciens \\
\hline 141 & MgAtr5 & PEG \\
\hline 237 & MgAtr5 & PEG \\
\hline
\end{tabular}

${ }^{\text {a }}$ Wild-type isolate.

b Transformation control with an ectopic integration of the hygromycinresistance cassette.

${ }^{\mathrm{c}}$ Agrobacterium tumefaciens-mediated transformation (Zwiers and De Waard 2001). Transformation strains are gene disruption mutants.

${ }^{\mathrm{d}}$ Polyethylene glycol (PEG)-mediated transformation (Payne et al. 1998; Zwiers et al, in press). Transformation strains are gene replacement mutants. pared with that of control strains IPO323 and Sp2 on wheat seedlings of the susceptible cultivar Obelisk. For all strains, first symptoms became visible at 8 to $10 \mathrm{dpi}$. These emerged as small chlorotic spots, most frequently starting from the tip of the leaves. These symptoms expanded longitudinally across the leaf surface and developed into necrotic lesions covered with pycnidia of the fungus. No difference in symptom development and necrotic leaf area was observed for strains IPO323, Sp2, and the disruption or replacement strains of MgAtr1, MgAtr2, MgAtr3, and MgAtr5 at $21 \mathrm{dpi}$. However, for the MgAtr4 disruption strains ( $\triangle \mathrm{MgAtr} 4.3$ and $\triangle \mathrm{MgAtr} 4.4$ ), symptom development was delayed, and lesions remained limited in size. At $21 \mathrm{dpi}, 50 \%$ of the leaf area from plants infected by the MgAtr4 disruption strains did not show symptoms, while leaves infected by the control strains were necrotic (Fig. 1). Lesions caused by the MgAtr4 disruption strains slowly continued to expand in time, and plants became necrotic at 30 to 35 dpi. Both MgAtr4 disruption strains tested showed the same phenotype.

None of the strains tested was impaired in pycnidia formation. Small necrotic lesions caused by the MgAtr4 disruption strains showed abundant pycnidia. Pycnidiospores isolated from plants inoculated with the MgAtr4 disruption strains were still able to grow on potato dextrose agar plates amended with hygromycin (100 mg per liter). Plants inoculated with these pycnidiospores showed the same reduction in virulence as plants inoculated with in vitro-grown yeast-like cells of the strains.

Virulence of strains $\Delta \mathrm{MgAtr} 4.3$ and $\Delta \mathrm{MgAtr} 4.4$ was also assessed on the wheat cultivars Taichung 29 and Bussard (susceptible to IPO323), Clement (semi-susceptible to IPO323), and Shafir and Toropi (resistant to IPO323). Both MgAtr4 disruption strains showed reduced virulence on all cultivars tested in comparison with that on the control strains. Production of pycnidia within necrotic areas was similar for all strains on all cultivars tested.

\section{In planta growth monitoring.}

Histopathological analysis of the infection process was examined by staining wheat leaves inoculated with control strain Sp2 and the MgAtr4 disruption strain $\Delta \mathrm{MgAtr} 4.3$ with trypan blue (Table 2). Germinated yeast-like cells showed germ tubes from both apical ends, and no signs of growth inhibition on the leaf surface were present for any of the strains tested. Chemo- or thigmotropism towards stomata was not observed, as in many cases, germ tubes by-passed guard cells of stomata without establishing infections. Infections appeared to occur only through stomata, as direct penetration of epidermal cells was not observed.

The control strain Sp2 had successfully entered and colonized the substomatal cavities by 6 dpi. Infection hyphae extended longitudinally toward neighboring substomatal cavities, establishing multiple infections from a single penetration site. At $10 \mathrm{dpi}$, the first macroscopical symptoms became visible on infected plants as chlorotic spots at the tip of the leaves (Fig. 2a). At this timepoint, microscopical observations showed strict intercellular growth of the fungus between mesophyll cells (Fig. 2b and c). Colonization of the leaf apoplast and increase of fungal biomass progressed rapidly from 10 dpi onward, and mycelial proliferation around substomatal cavities became apparent by 14 dpi. Collapse of leaf tissue and necrosis described the macroscopical symptoms at $17 \mathrm{dpi}$ (Fig. 2g). This coincided with abundant hyphal growth around mesophyll cells (Fig. 2h) and the formation of mycelial baskets in the substomatal cavities (Fig. 2i), followed by the development of pycnidia. At $21 \mathrm{dpi}$, leaves were almost completely necrotic (Fig. 2m). In dead leaf 
tissue, pycnidia were commonly present and coincided with extensive colonization of the apoplast (Fig. 2n and o).

Germ tubes of $\Delta \mathrm{MgAtr} 4.3$ penetrated the substomatal cavities at the same rate as in $\mathrm{Sp} 2$ but colonized substomatal cavities significantly slower. Growth of $\Delta \mathrm{MgAtr} 4.3$ towards the apoplast was also restricted. As for the control strain, however, chlorotic symptoms at the tip of the leaves became visible at 10 dpi (Fig. 2d), when fungal growth was still restricted to the substomatal cavities (Fig. 2e and f). To a limited extent, the fungus escaped infected substomatal cavities and grew longitudinally toward neighboring substomatal cavities, but the majority of established infections did not expand (Fig. 2k). Increase in fungal biomass in infected substomatal cavities and the apoplast progressed only slowly (14 and 17 dpi) (Fig. 2l). At $21 \mathrm{dpi}$, cell collapse was mainly observed in restricted necrotic lesions (Fig. 2p, q, and r), but pycnidia formation in these lesions progressed normally.

\section{Quantification of leaf colonization.}

Colonization of wheat leaves infected by control strain $\mathrm{Sp} 2$ and the MgAtr4 disruption strain $\Delta \mathrm{MgAtr} 4.3$ was quantified at 21 dpi by counting the number of colonized substomatal cavities, the abundance of biomass in the substomatal cavities, and the degree that adjacent substomatal cavities were colonized (systemic infection). In the latter case, the number of colonized substomatal cavities with either one or both of the two flanking substomatal cavities colonized was assessed. The total number of colonized substomatal cavities was significantly higher for Sp2 than for $\Delta \mathrm{MgAtr} 4.3$ (Table 3 ). Within necrotic lesions, biomass and pycnidia formation in colonized substomatal cavi-
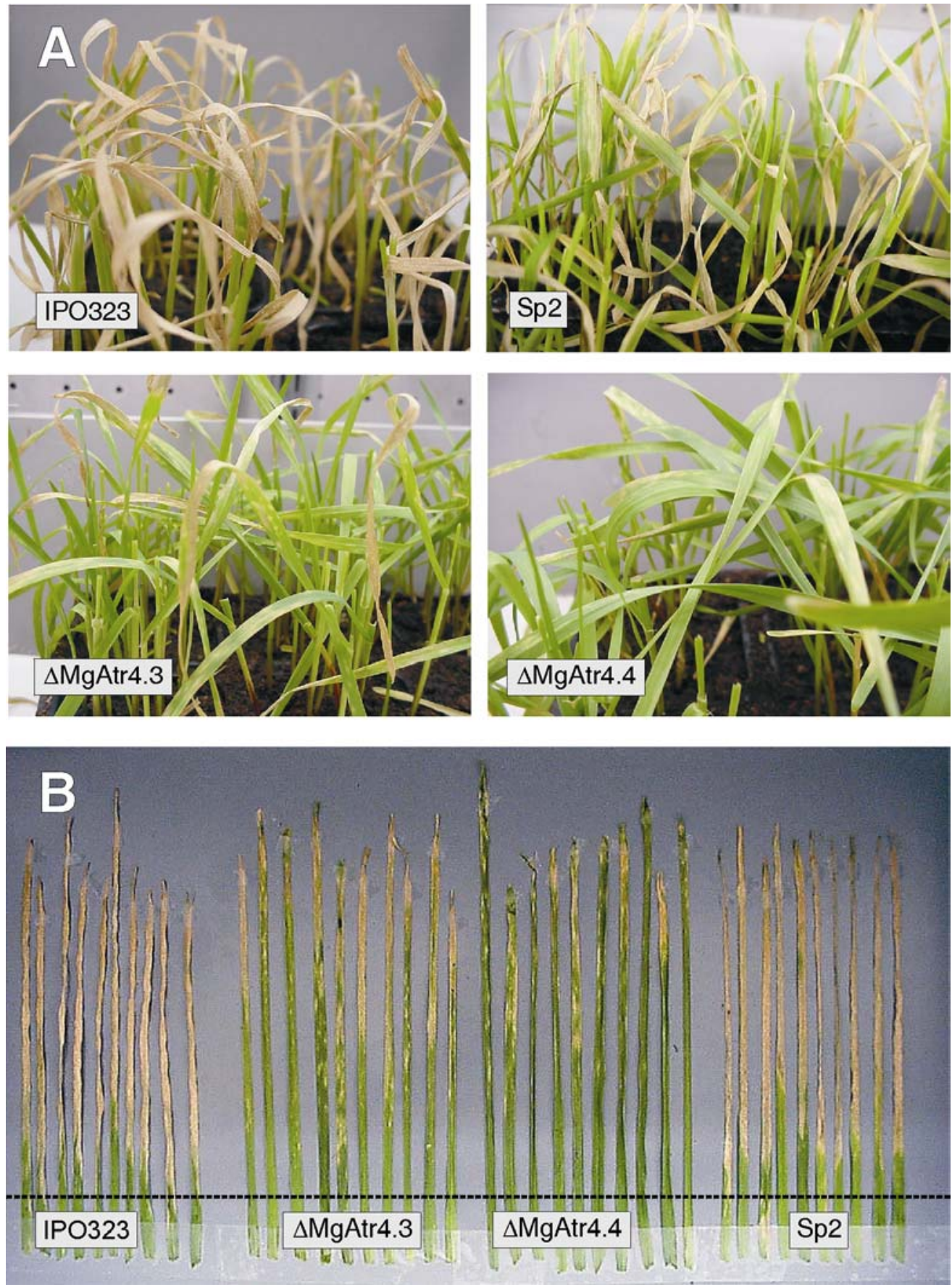

Fig. 1. Virulence of Mycosphaerella graminicola strains on wheat seedlings of cv. Obelisk at 21 days postinoculation. Strains tested: IPO323 and Sp2 (controls) and $\Delta \mathrm{MgAtr} 4.3$ and $\Delta \mathrm{MgAtr} 4.4$ (MgAtr4 disruption strains). A, Symptoms of whole plants. B, Symptoms on first emerging leaves. 
ties was similar for the two strains tested (Table 3). However, the systemic nature of substomatal colonization varied significantly between the two strains, since the percentage of flanking substomatal cavities that were colonized was much higher for strain Sp2 than for strain $\Delta \mathrm{MgAtr} 4.3$ (Table 3 ). Thus, the results indicate that the $M g A t r 4$ disruption strains possess a reduced ability to colonize substomatal cavities and expand into the mesophyll.

\section{In vitro growth.}

The effect of disruption of MgAtr4 on fitness of the fungus was studied in in vitro growth test experiments. Growth of strains $\Delta \mathrm{MgAtr} 4.3$ and $\Delta \mathrm{MgAtr} 4.4$ was compared with that of control strain $\mathrm{Sp} 2$ in liquid culture media that promote growth of the fungus as either yeast-like cells (yeast-sucrose medium [YSM], minimal medium (M-1-D), intercellular washing fluid from uninfected wheat leaves (IWF), and wheat leaves inoculated by $M$. graminicola strain IPO323 (IWF-IPO323), or mycelium (Czapek-Dox + mycolobical peptone [CzDMP]). Yeastlike growth of $\Delta \mathrm{MgAtr} 4.3$ and $\Delta \mathrm{MgAtr} 4.4$ was similar to that of Sp2 in all media tested (Fig. 3A through D). Growth in these media appeared to be exponential between 2 and 5 days of culturing, except for M-1-D. In the latter medium, exponential growth was still observed after 14 days of culturing. Biomass levels at the stationary phase were highest in YSM and significantly lower in IWF and IWF-IPO323. Mycelial growth in CzDMP was also similar for all isolates tested (Fig. 3E).

\section{Expression analysis.}

Expression of the ABC transporter genes MgAtrl, MgAtr2, $M g A t r 3, M g A t r 4$, and MgAtr5 and the fungal-specific CYP51 gene, encoding cytochrome P450 sterol $14 \alpha$-demethylase $\left(\mathrm{P} 450_{14 \mathrm{DM}}\right.$ ) (Kalb et al. 1987) from M. graminicola, was examined in interaction RNA isolated from wheat seedlings inoculated with the wild-type strain IPO323 and strains $\triangle \mathrm{MgAtr} 4.3$ and $\Delta \mathrm{MgAtr} 4.4$ in timecourse experiments. In all cases, expression of MgAtr2 and MgAtr3 was not detected. Low transcript levels of MgAtrl and MgAtr5 were observed at 22 dpi in interaction RNA isolated from plants inoculated with IPO323 (data not shown). Transcripts of MgAtr4 and CYP51 became visible at $12 \mathrm{dpi}$, and their levels gradually increased until 18 dpi. At 22 dpi, a small decrease in the transcript level of these genes was observed (Fig. 4). Transcripts of MgAtr4, CYP51, and the other genes tested could not be detected at any timepoint after infection in interaction RNA isolated from plants inoculated with the MgAtr4 disruption strains (Fig. 4).

\section{Phytotoxic activity of culture filtrates.}

Histopathological studies suggested the involvement of toxins in the wheat $-M$. graminicola interaction (Kema et al. 1996). Perrone and associates (1999) reported the production of a noncultivar-specific phytotoxic metabolite by M. graminicola in liquid M-1-D medium. Therefore, the phytotoxic activity of M-1-D culture filtrates from strains IPO323, Sp2, $\Delta \mathrm{MgAtr} 4.3$, and $\Delta \mathrm{MgAtr} 4.4$ was examined on wheat seedlings of cvs. Obelisk, Bussard (susceptible to IPO323), and Shafir (resistant to IPO323). Fourteen-day-old culture filtrates from these strains showed phytotoxic activity on all cultivars tested. Symptoms incited by all strains consisted of chlorotic spots that became visible at six to eight days postspraying (data not shown). These symptoms resembled the initial chlorotic spots observed upon infection with the fungus. Phytotoxicity of all culture filtrates was slightly higher to cv. Shafir than to cvs. Obelisk and Bussard. However, no difference in phytotoxic activity between the culture filtrates from strains IPO323, Sp2, $\Delta \mathrm{MgAtr} 4.3$, and $\Delta \mathrm{MgAtr} 4.4$ was observed on any of the wheat cultivars tested.

\section{DISCUSSION}

Our studies demonstrated that MgAtr4 disruption strains have a reduced ability to cause septoria tritici blotch on wheat seedlings in comparison with that of the wild-type control strains and the other $M g A t r$ disruption or replacement strains tested. Thus, MgAtr4 can be regarded as a virulence factor of M. graminicola on wheat, as defined by Shaner and associates (1992). MgAtr4 is the first virulence factor identified in this important plant pathogen.

MgAtr4 disruption strains have a normal in vitro growth, both as yeast-like cells and mycelium, and therefore, the delayed in planta growth of these strains can not be ascribed to a general fitness penalty. Histopathological analysis of the infection processes revealed that the reduced virulence of the MgAtr4 disruption strains can be ascribed to unsuccessful colonization of substomatal cavities. This early stage in pathogenesis is essential for establishment of the disease, further expansion of the fungus to the apoplast, and colonization of neighboring substomatal cavities, thereby allowing multiple stomatal infections from a single penetration site (Duncan and Howard 2000). Retarded growth of the MgAtr4 disruption strains in the apoplast may be the result of hampered growth in substomatal cavities.

Expression analysis of interaction RNA isolated from wheat inoculated with strain IPO323 showed increased transcript levels of MgAtr4 over time. A similar increase in transcript levels was observed for the constitutively expressed CYP51 gene from M. graminicola (Gisi et al. 2000). The increase in transcript levels of these two genes during infection correlated with an increase in fungal biomass characteristic for compatible interactions (Kema et al. 1996). In contrast, no transcripts of CYP51 were detected in interaction RNA of the MgAtr4 disruption strains, indicating that biomass proliferation of these strains during infection was extremely low. Hence, results from the expression analysis corroborate the finding of reduced in planta growth of the MgAtr4 disruption strains.

In plant pathogenic fungi, $\mathrm{ABC}$ transporters could act as virulence factors if they provide protection against plant defense compounds during pathogenesis or mediate secretion of pathogenicity factors, such as host-specific toxins or other virulence factors (De Waard 1997; Stergiopoulos et al. 2002b). The involvement of phytoalexins in the wheat $-M$. graminicola

Table 2. Histopathological analysis of the stages of pathogenesis by control strain Sp2 and the MgAtr4 disruption strain $\Delta \mathrm{MgAtr} 4.3$ of Mycosphaerella graminicola ${ }^{\mathrm{a}}$

\begin{tabular}{|c|c|c|c|c|c|c|c|c|}
\hline \multirow[b]{2}{*}{ Strains } & \multicolumn{2}{|c|}{ Leaf surface } & \multicolumn{2}{|r|}{ Stomatal infections } & \multicolumn{2}{|c|}{ Colonization of apoplast } & \multicolumn{2}{|c|}{ Symptoms } \\
\hline & $\begin{array}{c}\text { Germination } \\
1 \mathbf{d p \mathbf { p i } ^ { \mathbf { b } }}\end{array}$ & $\begin{array}{l}\text { Growth } \\
1-2 \text { dpi }\end{array}$ & $\begin{array}{l}\text { Entry } \\
1-2 \text { dpi }\end{array}$ & $\begin{array}{c}\text { Substomatal colonization } \\
\text { 3-6 dpi }\end{array}$ & $\begin{array}{l}\text { Longitudinal growth } \\
6-10 \mathrm{dpi}\end{array}$ & $\begin{array}{c}\text { Biomass build-up } \\
\text { 10-21 dpi }\end{array}$ & $\begin{array}{l}\text { Necrosis } \\
21 \text { dpi }\end{array}$ & $\begin{array}{c}\text { Pycnidia } \\
21 \text { dpi }\end{array}$ \\
\hline $\mathrm{Sp} 2$ & $++^{\mathrm{c}}$ & ++ & ++ & ++ & ++ & ++ & ++ & ++ \\
\hline$\Delta \mathrm{MgAtr} 4.3$ & ++ & ++ & ++ & + & + & + & + & + \\
\hline
\end{tabular}

\footnotetext{
${ }^{a}$ Table depicts differences between the two strains at various stages of infection.

${ }^{\mathrm{b}}$ Days postinoculation (dpi).

${ }^{\mathrm{c}}$ The + signs represent an arbitrary comparison between the two strains.
} 
interaction has been proposed, and phytoalexin detoxification might be essential for virulence (Kema et al. 1996). In that respect, accumulation of autofluorescent compounds around substomatal cavities in response to attack by M. graminicola, may be indicative for phytoalexin production (Cohen and Eyal 1993). Autofluorescent compounds do not completely arrest infection but rather affect the rate of in planta mycelial development at the onset of the infection (Pnini-Cohen et al. 1997). The autofluorescence around stomata in response to penetration by $M$. graminicola is more intense in resistant wheat cultivars than in susceptible ones, suggesting that autofluorescent compounds also contribute to resistance (Shetty and Jørgensen 2001). Therefore, it is possible that MgAtr4 is involved in protection of the fungus against fungitoxic compounds produced at the substomatal cavities. This would explain the decreased potency of the MgAtr4 disruption strains to colonize substomatal cavities and the retarded growth of these strains in the apoplast.

MgAtr4 is highly homologous to ABC1, an ABC transporter from Magnaporthe grisea that determines pathogenicity of this fungus on rice and barley (Urban et al. 1999). $A B C 1$ deletion mutants show a dramatic growth arrest after penetration of the host and fail to produce extensive infection hyphae and to colonize the apoplast. Although the exact role of $\mathrm{ABC} 1$ during pathogenesis is not known, the most probable explanation for the loss of virulence of the deletion mutants is that $\mathrm{ABC} 1$ provides protection against antimicrobial compounds produced by the host. The high homology between MgAtr4 and ABC1 and the similarities in the observed phenotype in disruption mutants of both transporter genes suggest that the encoded proteins may have a similar function during pathogenesis, possibly by providing protection against plant defense compounds produced by the host. Such a function in virulence has previously been demonstrated for the $\mathrm{ABC}$ transporter BcatrB from Botrytis cinerea (Schoonbeek et al. 2001) and GpABC1 from Gibberella pulicaris (Fleissner et al. 2002), which provide protection against the plant-defense compounds resveratrol and rishitin, respectively.

Heterologous expression of MgAtr1, MgAtr2, MgAtr4, and $M g A t r 5$ from $M$. graminicola in a multiple-ABC transporter knockout strain of $S$. cerevisiae showed that the encoded ABC transporters transport a wide variety of chemically unrelated compounds and possess an overlap in substrate specificity (Zwiers et al., in press). Therefore, the presence of functional homologues of MgAtr4 in M. graminicola may explain why MgAtr4 disruption strains do not show a complete loss of virulence on host plants.

Formation of necrotic lesions caused by M. graminicola may be associated with secretion of phytotoxic compounds by the pathogen (Kema et al. 1996). However, a function of MgAtr4 in secretion of phytotoxic metabolites could not be established, since disruption of MgAtr4 did not alter the phytotoxic activity of culture filtrates. A role in self-protection to endogenous toxins is also not likely, since in vitro growth of MgAtr4 disruption strains under conditions that promote the production of such metabolites was not impaired.

\section{MATERIALS AND METHODS}

Strains, media, and growth conditions.

M. graminicola isolate IPO323 (Kema and Van Silfhout 1997) was used as a wild-type control and as recipient strain for the generation of disruption or replacement strains. Singlegene disruption strains of $M g A t r 1, M g A t r 2$, and $M g A t r 4$ were generated using Agrobacterium tumefaciens-mediated transformation (Zwiers and De Waard 2001; Zwiers et al., in press). Gene replacement strains of MgAtr3 and MgAtr5 were made according to Payne and associates (1998) and Zwiers and associates (2002). Strain Sp2 is a control transformant containing a single copy of the hygromycin-resistance cassette generated by A. tumefaciens-mediated transformation (Table 1).

Yeast-like cells were grown in liquid YSM (10 g of yeast extract and $10 \mathrm{~g}$ of sucrose per liter) in the dark at $18^{\circ} \mathrm{C}$ and were shaken at $140 \mathrm{rpm}$. Cultures $(20 \mathrm{ml})$ were inoculated with yeast-like cells derived from stocks stored at $-80^{\circ} \mathrm{C}$. The optical density of cultures was determined at $600 \mathrm{~nm}\left(\mathrm{OD}_{600}\right)$ and new starting cultures $(20 \mathrm{ml})$ were prepared with an $\mathrm{OD}_{600}$ of 0.05 . Three days later, the $\mathrm{OD}_{600}$ of these cultures was adjusted to 0.5 in fresh medium $(20 \mathrm{ml})$, and the cultures were incubated again overnight under conditions similar to those described above. In all cases the antibiotic streptomycin sulfate (100 mg per liter) was added to the cultures to prevent bacterial growth.

\section{Virulence assays.}

Virulence of M. graminicola strains was tested on the wheat cultivar Obelisk, which is susceptible to isolate IPO323. For each MgAtr gene, two independent disruption or deletion strains were tested (Table 3), and virulence assays were repeated at least three times. M. graminicola yeast-like cells grown in liquid YSM were harvested by centrifugation at 3,000 $\times \mathrm{g}$ for $10 \mathrm{~min}$ at $10^{\circ} \mathrm{C}$. Pellets were washed once in sterile Millique water and were resuspended in $0.15 \%$ Tween 20 at a density of $10^{7}$ cells per ml. Suspensions were sprayed onto seven- to nine-day-old wheat seedlings until run-off. Control treatments were performed with a solution of $0.15 \%$ Tween 20 . Inoculated plants were placed in sealed perspex-lidded containers at $18^{\circ} \mathrm{C}$ and a 16-h-daylight period. Relative humidity ( $\left.\mathrm{RH}\right)$ in the containers was kept above $80 \%$ by placing a watersoaked cloth in the bottom of the boxes. Emerging second leaves were clipped every four to five days, to facilitate disease assessment and light penetration to the inoculated first emerg-

Table 3. Quantification of infection of Mycosphaerella graminicola Sp2 (control strain) and $\triangle \mathrm{MgAtr} 4.3$ (MgAtr4 disruption strain) on wheat leaves of cv. Obelisk at 21 days postinoculation

\begin{tabular}{|c|c|c|c|c|}
\hline \multirow[b]{2}{*}{ Substomatal cavities } & \multicolumn{2}{|c|}{ Sp2 } & \multicolumn{2}{|c|}{$\Delta$ MgAtr4.3 } \\
\hline & Number & $\%$ & Number & $\%$ \\
\hline Colonized & 2205 & 44 & 1066 & 21 \\
\hline Noncolonized & 2795 & 56 & 3934 & 79 \\
\hline Presence of mycelial biomass at level $1^{\mathrm{a}}$ & 1516 & 69 & 740 & 69 \\
\hline Presence of mycelial biomass at level 2 & 689 & 31 & 326 & 31 \\
\hline Pattern of colonization: $0-1-0^{\mathrm{b}}$ & 298 & 13.5 & 423 & 40 \\
\hline Pattern of colonization: $0-1-1$ or $1-1-0$ & 674 & 30.5 & 332 & 31 \\
\hline Pattern of colonization: $1-1-1$ & 1233 & 56.0 & 311 & 29 \\
\hline
\end{tabular}

\footnotetext{
${ }^{a}$ Presence of biomass in the colonized substomatal cavities. Level 1, limited mycelium; level 2, mycelial basket or pycnidium.

${ }^{\mathrm{b}}$ Numbers indicate patterns of colonization of two substomatal cavities (first and third figure) flanking a colonized substomatal cavity (middle figure). 0 indicates uncolonized substomatal cavities, and 1 indicates colonized substomatal cavities.
} 

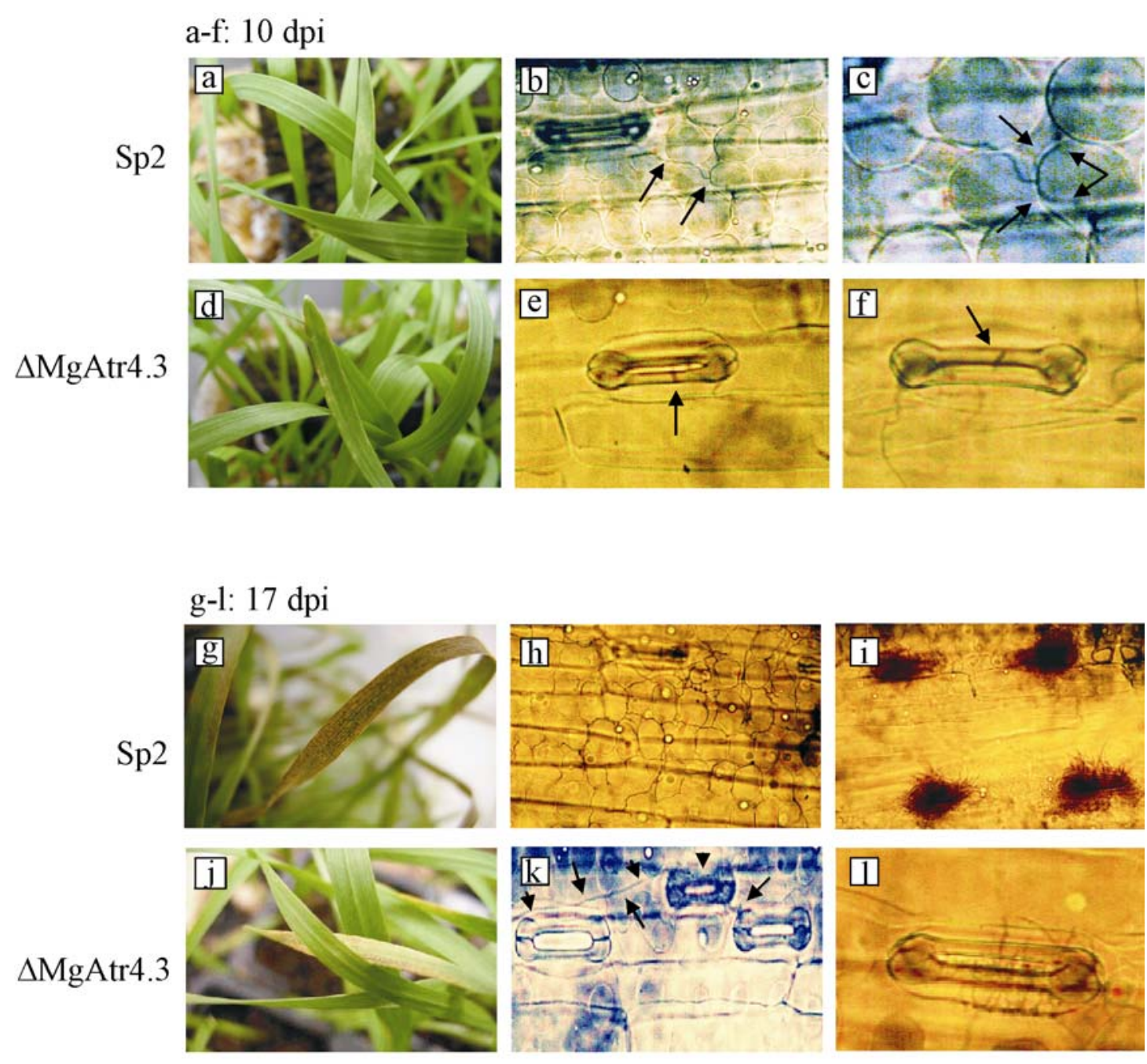

m-r: 21 dpi

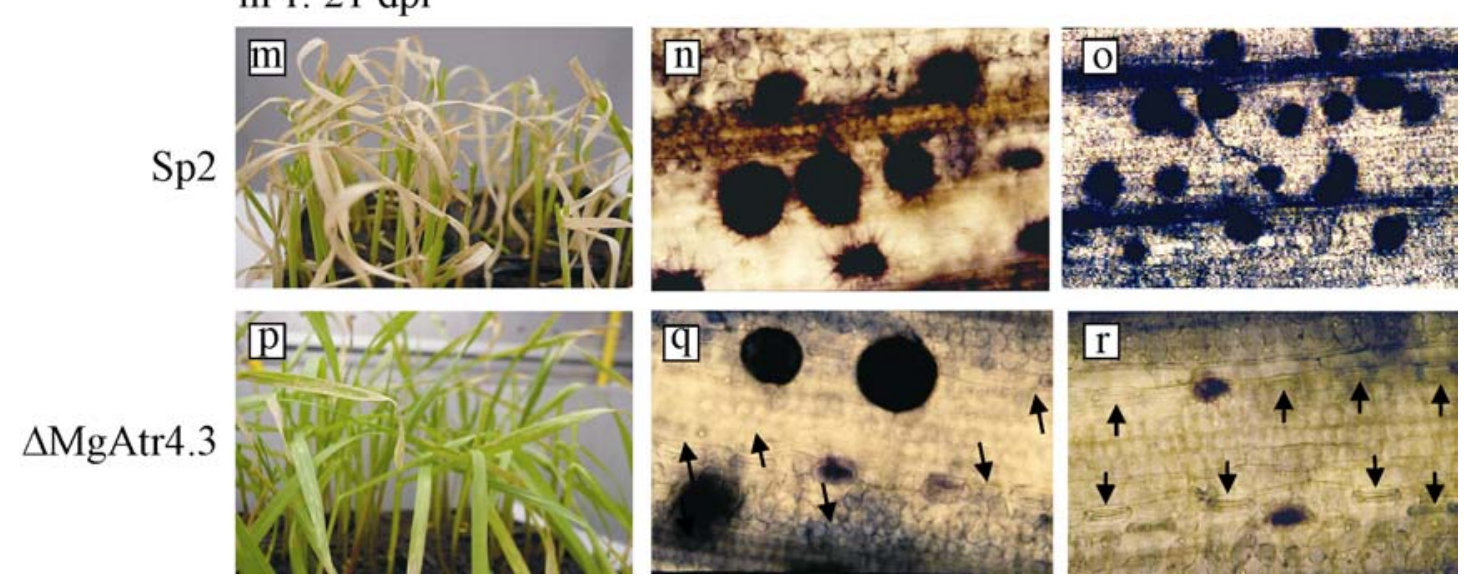

Fig. 2. Reduced in planta growth of strain $\triangle \mathrm{MgAtr} 4.3$ (MgAtr4 disruption strain) of Mycosphaerella graminicola in comparison with strain Sp2 (control) over time. Panels a to $\mathbf{f}$ show growth at $10 \mathrm{dpi}$. a, macroscopical symptoms incited by $\mathrm{Sp} 2$; $\mathbf{b}$, intercellular growth to the apoplast (arrows); $\mathbf{c}$, detail of $\mathbf{b}$ showing infection hyphae growing around mesophyll cells (arrows); d, macroscopical symptoms incited by $\Delta \mathrm{MgAtr} 4.3$; e, infection hyphae restricted at the substomatal cavity (arrow); and $\mathbf{f}$, same as e. Panels $\mathbf{g}$ to $\mathbf{l}$ show growth at $17 \mathrm{dpi}$. $\mathbf{g}$, macroscopical symptoms incited by Sp2; h, extensive mycelial growth in the apoplast; $\mathbf{i}$, mycelial baskets in substomatal cavities (level 2); $\mathbf{j}$, macroscopical symptoms incited by $\Delta \mathrm{MgAtr} 4.3$; $\mathbf{k}$, longitudinal growth to neighboring substomatal cavities without basket formation in substomatal cavities (arrows); and l, limited mycelial biomass in substomatal cavities (level 1). Panels $\mathbf{m}$ to $\mathbf{r}$ show growth at 21 dpi. $\mathbf{m}$, macroscopical symptoms incited by Sp2; $\mathbf{n}$, pycnidia in necrotic lesions; $\mathbf{o}$, same as $\mathrm{n}$; $\mathbf{p}$, macroscopical symptoms incited by $\Delta \mathrm{MgAtr} 4.3 ; \mathbf{q}$, stomata with pycnidia and uninfected stomata (arrows); and $\mathbf{r}$, scattered infected stomata on the leaf surface (arrows indicate uninfected stomata). 

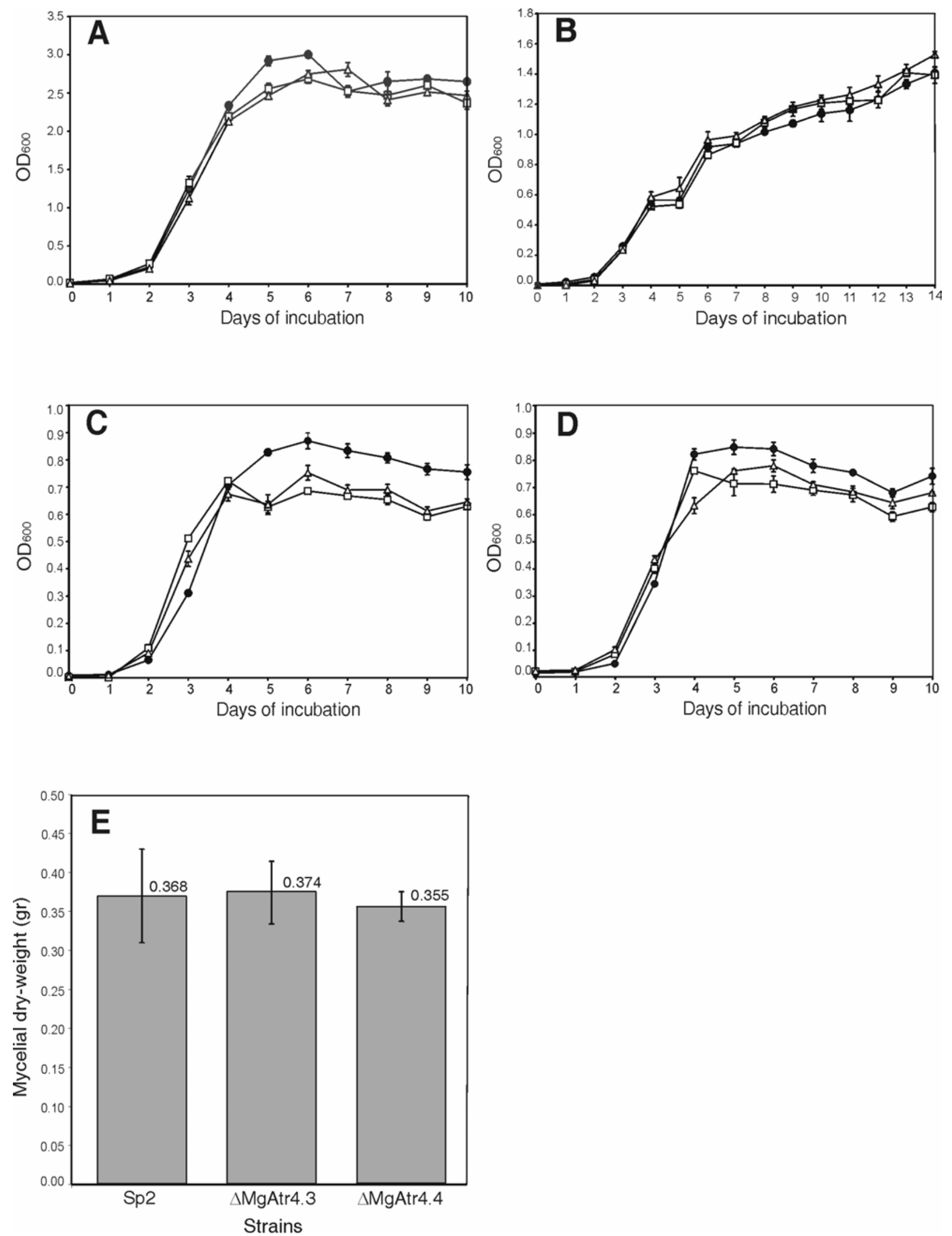

Fig. 3. In vitro growth of Mycosphaerella graminicola strains $\mathrm{Sp} 2(\bullet)$ (control), $\Delta \mathrm{MgAtr} 4.3(\square)$, and $\Delta \mathrm{MgAtr} 4.4(\Delta)($ MgAtr4 disruption strains) in liquid media that promote yeast-like growth (A through $\mathbf{D})$ or mycelium $(\mathbf{E})$ : A, Yeast sucrose medium, B, M-1-D minimal medium, $\mathbf{C}$, intercellular washing fluid, and D, intercellular washing fluid isolated from plants infected by the wild-type M. graminicola isolate IPO323, and $\mathbf{E}$, Czapek-Dox + mycological peptone medium. Yeast-like growth was assessed by measuring the optical density of cultures at $600 \mathrm{~nm}\left(\mathrm{OD}_{600}\right)$. Mycelial growth was assessed by dry weight measurments. 
ing leaf. Virulence was assessed visually by evaluation of the leaf area covered with symptoms and the abundance of pycnidia in necrotic lesions.

In addition to cv. Obelisk, virulence of the MgAtr4 disruption strains was also assessed on cvs. Taichung 29, Bussard, Clement, Shafir, and Toropi. The response of these cultivars to infections with IPO323 varied from susceptible (Taichung 29, Bussard) to semi-susceptible (Clement) and to resistant (Shafir, Toropi).

\section{Histopathological analysis.}

Wheat seedlings of cv. Obelisk were inoculated with strains Sp2 and $\Delta \mathrm{MgAtr} 4.3$, as previously described. Entire leaves ( $n$ $=5$ ) were collected from inoculated plants at 3, 6, 10, 14, 17, and 21 dpi and were stained with trypan blue (Heath 1971; Wilson and Coffey 1980) to monitor fungal growth inside leaf tissue. Stained leaves were examined with a Zeiss Axiovert 10 microscope (Zeiss, Jena, Germany), and photographs were made on a $35 \mathrm{~mm}$ Kodak Ektachrome 64T Color Reversal Film (Kodak, Herts, England).

Quantitative assessment of disease caused by strains Sp2 and $\Delta \mathrm{MgAtr} 4.3$ was made at $21 \mathrm{dpi}$, by counting the number of colonized substomatal cavities present in the two parallel rows adjacent to the central vein on one side of the leaves. Counting started from the tip of the leaves, and the first 500 substomatal cavities from each row were used for calculations (in total 5,000 for each interaction). Assessment of fungal biomass in colonized substomatal cavities was performed by scoring the presence of limited mycelial growth (level 1) or the presence of a mycelial basket or a mature pycnidium at the substomatal cavities (level 2). The systemic nature of substomatal colonization by strains $\mathrm{Sp} 2$ and $\Delta \mathrm{MgAtr} 4.3$ was calculated by counting the number of colonized substomatal cavities flanking a colonized substomatal cavity.

\section{In vitro growth experiments.}

Growth of yeast-like cells and mycelium of strain Sp2 was compared with growth of the MgAtr4 disruption strains $\triangle \mathrm{MgAtr} 4.3$ and $\Delta \mathrm{MgAtr} 4.4$, in liquid culture media.

Media used for yeast-like cells were YSM, M-1-D, and IWF isolated from uninoculated and IPO323-inoculated (IWFIPO323) wheat seedlings. M-1-D is a defined minimal medium containing sucrose $(87.6 \mathrm{mM}), \mathrm{MgSO}_{4}(30 \mathrm{mM})$, ammonium tartrate $(27.1 \mathrm{mM}), \mathrm{Ca}\left(\mathrm{NO}_{3}\right)_{2}(1.2 \mathrm{mM}), \mathrm{KNO}_{3}(0.79 \mathrm{mM})$, $\mathrm{KCl}(0.87 \mathrm{mM}), \mathrm{NaH}_{2} \mathrm{PO}_{4}(0.14 \mathrm{mM}), \mathrm{KI}(45 \mu \mathrm{M}), \mathrm{H}_{3} \mathrm{BO}_{3}(22$ $\mu \mathrm{M}), \mathrm{MnSO}_{4}(30 \mu \mathrm{M}), \mathrm{ZnSO}_{4}(8.7 \mu \mathrm{M})$, and $\mathrm{FeCl}_{2} 6 \mathrm{H}_{2} \mathrm{O}(7.4$ $\mu \mathrm{M})$ (Pinkerton and Strobel 1976). IWF was isolated from wheat seedlings grown for 10 to 12 days at $18^{\circ} \mathrm{C}$ and $\mathrm{RH}$ above $80 \%$ by water infiltration in vacuo, followed by centrifugation at 3,000 $\times \mathrm{g}(\mathrm{De}$ Wit and Spikman 1982). IWF from wheat seedlings inoculated with IPO323 was isolated according to the same procedure from leaves inoculated 8 days post sowing and harvested at $4 \mathrm{dpi}$. The IWFs were concentrated by lyophilization to half of their original volume before use.

Yeast-like cells of the strains used in growth-test experiments were precultured for 3 days in liquid YSM, as described previously in this paper. Growth was assessed by measuring the $\mathrm{OD}_{600}$ in cuvettes with a 1-cm optical path length with a BioPhotometer (Eppendorf, Hamburg, Germany). New cultures $(25 \mathrm{ml})$ with an $\mathrm{OD}_{600}$ of 0.01 were initiated in the media described above and were incubated in the dark at $18^{\circ} \mathrm{C}$ and $140 \mathrm{rpm}$. Samples $(n=5)$ were taken daily for a period of 10 (YSM, IWF, and IWF-IPO323) or 14 days (M-1-D). The $\mathrm{OD}_{600}$ of these samples was corrected with the $\mathrm{OD}_{600}$ values of the culture filtrates obtained after centrifugation at $10,000 \times g$ for 5 min.

Mycelial growth of the strains was tested in Czapek-Dox + mycological peptone (CzDMP) (Czapek-Dox, $33.4 \mathrm{~g}$ per liter; mycological peptone, $5 \mathrm{~g}$ per liter). Yeast-like cells were precultured for 3 days in liquid YSM, as described previously in this paper. These cells were used to initiate in 10-fold new cultures in CzDMP $(20 \mathrm{ml})$ with a starting density of $10^{5}$ spores per $\mathrm{ml}$ and were incubated in the dark at $25^{\circ} \mathrm{C}$ and $140 \mathrm{rpm}$ for 5 days. Then mycelial biomass was harvested by centrifugation $(10,000 \times g, 10 \mathrm{~min})$ and was lyophilized for $72 \mathrm{~h}$. Growth of the strains was assessed by dry weight measurements $(n=10)$.

\section{Interaction RNA and Northern blot analysis.}

Northern analysis was performed with total RNA isolated from infected wheat leaves in timecourse experiments. Seedlings of cv. Obelisk (9 day old) were inoculated with isolate IPO323 and strains $\Delta \mathrm{MgAtr} 4.3$ and $\Delta \mathrm{MgAtr} 4.4$. Control inoculations were performed with a solution of $0.15 \%$ Tween 20 . First emerging leaves $(n=5)$ were collected over a period of twenty-two days at $6,8,12,14,16,18$, and $22 \mathrm{dpi}$, were instantly frozen in liquid nitrogen, and were stored at $-80^{\circ} \mathrm{C}$ until RNA isolation.
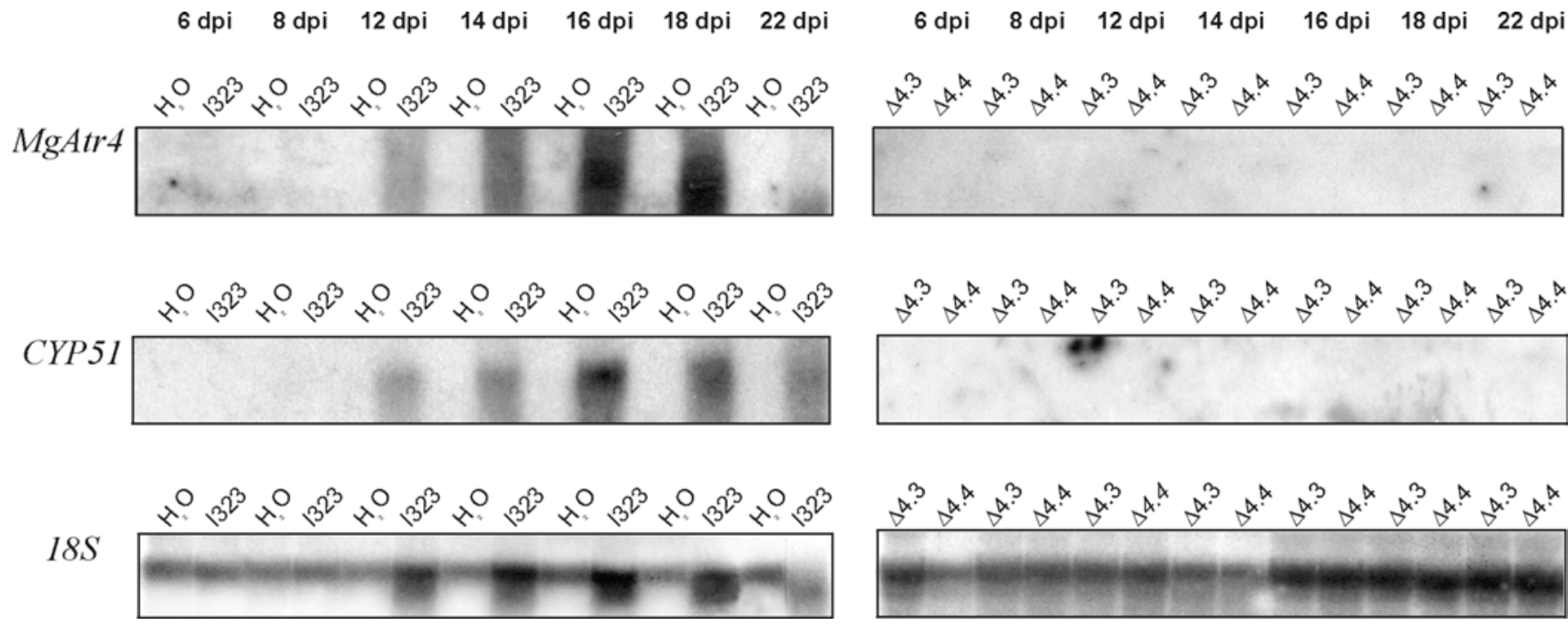

Fig. 4. Expression analysis of MgAtr4 and CYP51 from Mycosphaerella graminicola in interaction RNA isolated from leaves of wheat seedlings inoculated with the wild-type strain IPO323 (I323) and the MgAtr4 disruption strains $\Delta \mathrm{MgAtr} 4.3(\Delta 4.3)$ and $\Delta \mathrm{MgAtr} 4.4$ ( $\Delta 4.4)$ in a timecourse experiment. Control treatments are indicated as $\mathrm{H}_{2} \mathrm{O}$. 
Interaction RNA was isolated from frozen biomass using the TRIzol reagent (Life Technologies Inc., Gaithersburg, MD, U.S.A.) according to the manufacturer's instructions. Isolated RNA $(15 \mu \mathrm{g}, 4.5 \mu \mathrm{l})$ was denatured in a solution of 6 M glyoxal $(4.5 \mu \mathrm{l}), 0.1 \mathrm{M}$ sodium phosphate $(3.0 \mu \mathrm{l})$, and dimethyl sulfoxide $(13.3 \mu \mathrm{l})$ at $50^{\circ} \mathrm{C}$ for $1 \mathrm{~h}$. RNA was subjected to electrophoresis on a $1.6 \%$ agarose gel in a $10 \mathrm{mM}$ sodium phosphate buffer, $\mathrm{pH}$ 7.0. Blotting was carried out on Hybond $\mathrm{N}^{+}$membranes (Amersham Life Science, Buckinghamshire, U.K.) by capillary transfer with 10× SSC (1× SSC is $0.15 \mathrm{M} \mathrm{NaCl}$ plus $0.015 \mathrm{M}$ sodium citrate) solution. RNA was cross-linked to membranes by irradiation under UV light (0.6 J per $\mathrm{cm}^{2}$ ). Homologous hybridizations were performed overnight at $65^{\circ} \mathrm{C}$ in Nasmyth's solution buffer $(18.5 \%$ dextran sulphate, $1.85 \%$ sarcosyl, $0.011 \mathrm{M}$ EDTA, $0.3 \mathrm{M}$ $\mathrm{Na}_{2} \mathrm{HPO}_{4} 1.1 \mathrm{M} \mathrm{NaCl}, \mathrm{pH}$ 6.2). This solution $(5.4 \mathrm{ml})$ was mixed with distilled water $(4.6 \mathrm{ml})$ just before use, to obtain the hybridization buffer $(10 \mathrm{ml})$. Blots were washed twice in $2 \times$ SSC, $0.1 \times$ sodium dodecyl sulfate (SDS) and twice in $0.1 \times \mathrm{SSC}, 0.1 \times \mathrm{SDS}$ at $65^{\circ} \mathrm{C}$ for $15 \mathrm{~min}$.

\section{Probes.}

Probes used in Northern blot analysis were a 0.84-kb EcoRI fragment of MgAtr1, a $0.75-\mathrm{kb}$ SalI fragment of MgAtr2, a 0.85-kb SalI fragment of MgAtr3, a 0.48-kb BamHI/PstI fragment of MgAtr4, a 0.6-kb EcoRI fragment of MgAtr5, and a 0.65 -kb fragment of $C Y P 51$ from $M$. graminicola. This last fragment was obtained by polymerase chain reaction, using genomic DNA from isolate IPO323 as template. Equal loading of samples on blots was examined with a 0.6-kb EcoRI fragment of the $18 S$ rRNA gene from Aspergillus niger.

Randomly primed DNA isotopic probes were prepared by enzymatic incorporation of $\left[\alpha^{32} \mathrm{P}\right]$-dATP. In each labeling reaction, $50 \mathrm{ng}$ of a probe template was used. Probes were purified using the QIAquick nucleotide removal kit (Qiagen, Leusden, The Netherlands) before addition to the hybridization solution.

\section{Production of phytotoxic compounds.}

Production of phytotoxic compounds by $M$. graminicola was tested in liquid M-1-D medium, according to Perrone and associates (1999). Cultures $(100 \mathrm{ml})$ of strains IPO323, Sp2, $\triangle \mathrm{MgAtr} 4.3$, and $\Delta \mathrm{MgAtr} 4.4$ were initiated at a starting $\mathrm{OD}_{600}$ of 0.1 and were grown for a period of 14 days in the dark at $22^{\circ} \mathrm{C}$ and $150 \mathrm{rpm}$. Cell-free culture filtrates were obtained by centrifugation at $10,000 \times g$ at $4^{\circ} \mathrm{C}$ for $10 \mathrm{~min}$. The supernatant was concentrated to half of the original volume by lyophilization and was filter sterilized through a $0.2 \mu \mathrm{m}$ filter (FP 30/0.2 CA-S, Schleicher and Schuell, Dassel, Germany). Culture filtrates were supplemented with $0.2 \%$ (vol/vol) Tween 80 , and phytotoxic activity was examined on 10-day-old wheat seedlings of cvs. Obelisk, Bussard, and Shafir by spraying until run-off. Control treatments were performed with M-1-D medium supplemented with $0.2 \%$ (vol/vol) Tween 80 . Plants were incubated in sealed Perspex-lidded containers at $18^{\circ} \mathrm{C}$ with a 16-h-daylight period for 3 weeks. Phytotoxicity was assessed at the end of the three-week period.

\section{ACKNOWLEDGMENTS}

We acknowledge G. H. J. Kema, E. C. P. Verstappen, and C. Waalwijk (Plant Research International, Wageningen, The Netherlands) for kindly providing the $M$. graminicola isolate IPO323 and for helpful suggestions and discussions during this work within the Wageningen Mycosphaerella group. We also acknowledge P. J. G. M. De Wit for critically reading the manuscript. We thank M. Calivas and A. C. Prince for helpful contribution to the expression analysis and the in vitro growth test experiments. I. Stergiopoulos was supported financially by the Training and Mobility of
Researchers (TMR) Program-Marie Curie Research Training Grants of the European Commission (Contract No. ERBFMBICT983558) and L.H. Zwiers was supported by Syngenta, Switzerland.

\section{LITERATURE CITED}

Brading, P. A., Verstappen, E. C. P., Kema, G. H. J., and Brown, J. K. M. 2002. A gene-for-gene relationship between wheat and Mycosphae rella graminicola, the Septoria tritici blotch pathogen. Phytopathology 92:439-445.

Cohen, L., and Eyal, Z. 1993. The histology of processes associated with the infection of resistant and susceptible wheat cultivars with Septoria tritici. Plant Pathol. 42:737-743.

De Waard, M. A. 1997. Significance of ABC transporters in fungicide sensitivity and resistance. Pestic. Sci. 51:271-275.

De Wit, P. J. G. M., and Spikman, G. 1982. Evidence for the occurrence of race and cultivar-specific elicitors of necrosis in intercellular fluids of compatible interactions of Cladosporium fulvum and tomato. Physiol. Plant Pathol. 21:1-11.

Duncan, K. E., and Howard, R. J. 2000. Cytological analysis of wheat infection by the leaf blotch pathogen Mycosphaerella graminicola. Mycol. Res. 104:1074-1082.

Eyal, Z., Scharen, A. L., Prescott, J. M., and van Ginkel, M. 1987. The Septoria Diseases of Wheat: Concepts and Methods of Disease Management. International Maize and Wheat Improvement Center, Mexico City, Mexico.

Fleissner, A., Sopalla, C., and Weltring, K.-M. 2002. An ATP-binding cassette multidrug-resistance transporter is necessary for tolerance of Gibberella pulicaris to phytoalexins and virulence on potato tubers. Mol. Plant-Microbe Interact. 15:102-108.

Gisi, U., Chin, K. M., Knapova, G., Farber, R. K., Mohr, U., Parisi, S., Sierotzki, H., and Steinfeld, U. 2000. Recent developments in elucidating modes of resistance to phenylamide, DMI and strobilurin fungicides. Crop Prot. 19:863-872.

Heath, M. C. 1971. Haustorial sheath formation in cowpea leaves immune to rust infection. Phytopathology 61:383-388.

Higgins, C. F. 1992. ABC transporters: From microorganisms to man. Annu. Rev. Cell. Biol. 8:67-113.

Kalb, V. F., Woods, C. W., Turi, T. G., Dey, C. R., Sutter, T. R., and Loper, J. C. 1987. Primary structure of the P450 lanosterol demethylase gene from Saccharomyces cerevisiae. DNA 6:529-537.

Kema, G. H. J., and Van Silfhout, C. H. 1997. Genetic variation for virulence and resistance in the wheat-Mycosphaerella graminicola pathosystem III. Comparative seedling and adult plant experiments. Phytopathology 87:266-272.

Kema, G. H. J., Yu, D., Rijkenberg, F. H. J., Shaw, M. W., and Baayen, R. P. 1996. Histology of the pathogenesis of Mycosphaerella graminicola in wheat. Phytopathology 86:777-786.

Kema, G. H. J., Verstappen, E. C. P., and Waalwijk, C. 2000. Avirulence in the wheat Septoria tritici leaf blotch fungus Mycosphaerella graminicola is controlled by a single locus. Mol. Plant-Microbe Interact. 13:1375-1379.

Nelson, L. R., and Marshall, D. 1990. Breeding wheat for resistance to Septoria nodorum and Septoria tritici. Adv. Agron. 44:257-277.

Payne, A. C., GrosjeanCournoyer, M. C., and Hollomon, D. W. 1998. Transformation of the phytopathogen Mycosphaerella graminicola to carbendazim and hygromycin B resistance. Curr. Genet. 34 (2):100104.

Perrone, G., Logrieco, A., Kema, G. J. H., Ritieni, A., and Bottalico, A. 1999. Phytotoxic activity of Mycosphaerella graminicola culture filtrates. Pages 39-45 in: Vol. 6 Proceedings of Phytosfere 1999, Rome, Italy.

Pinkerton, F., and Strobel, G. 1976. Serinol as an activator of toxin production in attenuated cultures of Helminthosporium sacchari (Eyespot disease of sugarcane). Proc. Natl. Acad. Sci. U.S.A. 73:4007-4011.

Pnini-Cohen, S., Zilberstein, A., Schuster, S., Sharon, A., and Eyal, Z. 1997. The expression of the uidA reporter gene and virulence in transformants of Septoria tritici. Page 60 in: Proceedings of the 15th Long Ashton International Symposium on Understanding Pathosystems: A Focus on Septoria. Long Ashton, U.K.

Schoonbeek, H.-J., Del Sorbo, G., and De Waard, M. A. 2001. The ABC transporter BcatrB affects the sensitivity of Botrytis cinerea to the phytoalexin resveratrol and the fungicide fenpiclonil. Mol. Plant-Microbe Interact. 14:562-571.

Schoonbeek, H., Raaijmakers, J. M., and De Waard, M. A. 2002. Fungal $\mathrm{ABC}$ transporters and microbial interactions in natural environments. Mol. Plant-Microbe Interact. 15:1165-1172.

Shaner, G., Stromberg, E. L., Lacy, G. H., Barker, K. R., and Pirone, T. P. 1992. Nomenclature and concepts of pathogenicity and virulence. Annu. Rev. Phytopathol. 30:47-66. 
Shetty, N. P., and Jorgensen, N. J. L. 2001. Histochemical studies of defence responses of wheat against Septoria tritici. Abst. no. 433. Proceedings of the 10th International Congress on Molecular PlantMicrobe Interactions, Madison, WI, U.S.A.

Stergiopoulos, I., Gielkens, M. M., Goodall, S. D., Venema, K., and De Waard, M. A. 2002a. Molecular cloning and characterisation of three new ATP-binding cassette transporter genes from the wheat pathogen Mycosphaerella graminicola. Gene 289:141-149.

Stergiopoulos, I., Zwiers, L.-H., and De Waard M. A. 2002b. Secretion of natural and synthetic toxic compounds from filamentous fungi by membrane transporters of the ATP-binding cassette and major facilitator superfamily. Eur. J. Plant Pathol. 108:719-734.

Urban, M., Bhargava, T., and Hamer, J. E. 1999. An ATP-driven efflux pump is a novel pathogenicity factor in rice blast disease. EMBO (Eur.
Mol. Biol. Organ.) J. 18:512-521.

Wilson, U. E., and Coffey, M. D. 1980. Cytological evaluation of general resistance to Phytophthora infestans (late blight) in potato foliage. Ann. Bot. 45:81-90.

Zwiers, L.-H., and De Waard, M. A. 2000. Characterization of the ABC transporter genes $M g A t r 1$ and MgAtr2 from the wheat pathogen $M y$ cosphaerella graminicola. Fun. Gen. Biol. 30:115-125.

Zwiers, L.-H., and De Waard, M. A. 2001. Efficient Agrobacterium tumefaciens-mediated gene disruption in the phytopathogen Mycosphaerella graminicola. Curr. Genet. 39:388-393.

Zwiers, L. H., Stergiopoulos, I., Gielkens, M. M. C., Goodall, S. D., and De "Waard, M. A. ABC transporters of the wheat pathogen $M y$ cosphaerella graminicola function as protectants against biotic and xenobiotic compounds. Mol. Gen. Genet., in press. 